\title{
Article \\ Testing Smart Grid Scenarios with Small Volume Testbed and Flexible Power Inverter
}

\author{
Milosz Krysik (D), Krzysztof Piotrowski *(D) and Krzysztof Turchan
}

Citation: Krysik, M.; Piotrowski, K. Turchan, K. Testing Smart Grid Scenarios with Small Volume Testbed and Flexible Power Inverter. Energies 2022, 15, 428. https://doi.org/ $10.3390 /$ en15020428

Academic Editors: Pierluigi Siano, Hassan Haes Alhelou and Muhammad Aziz

Received: 26 November 2021

Accepted: 1 January 2022

Published: 7 January 2022

Publisher's Note: MDPI stays neutral with regard to jurisdictional claims in published maps and institutional affiliations.

Copyright: (C) 2022 by the authors. Licensee MDPI, Basel, Switzerland. This article is an open access article distributed under the terms and conditions of the Creative Commons Attribution (CC BY) license (https:// creativecommons.org/licenses/by/ $4.0 /)$.
IHP_Leibniz Institute for High Performance Microelectronics, 15236 Frankfurt, Germany; krysik@ihp-microelectronics.com (M.K.); turchan@ihp-microelectronics.com (K.T.)

* Correspondence: piotrowski@ihp-microelectronics.com

\begin{abstract}
The growing penetration of Renewable Energy Sources (RES) due to the transition to future smart grid requires a huge number of power converters that participate in the power flow. Each of these devices needs the use of a complex control and communication system, thus a platform for testing real-life scenarios is necessary. Several test techniques have been so far proposed that are subject to a trade-off between cost, test coverage, and test fidelity. This paper presents an approach for testing microgrids, by developing an emulator, with emphasis on the micro-inverter unit and the possibility of flexible configuration for different grid topologies. In contrast to other approaches, our testbed is characterized by small volume and significantly scaled-down voltages for safety purposes. The examination is concentrated specifically on the inverter behavior. The test scenarios include behaviors in case of load changes, transition between grid-tied and islanded mode, connection and removal of subsequent inverters, and prioritization of inverters.
\end{abstract}

Keywords: microgrid (MG); smart grid (SG); power hardware in the loop (PHIL); power inverter; microgrid emulator

\section{Introduction}

The world undeniably must turn from fossil fuels to a greener course to produce energy, for instance, with wind, solar, nuclear or bioenergy sources. An important organization in the transformation is the European Union (EU). The EU, as part of the European Green Deal, plans to target by 2030 at least 32.5\% improvement in energy efficiency, 32\% share for renewable energy, and 40\% cuts in greenhouse gas emissions (from 1990 levels) [1]. The European Council established in 2019 the objective of making the EU climate-neutral by 2050, in line with the Paris Agreement. The smart grid (SG) is a key element in the global trend of the future energy system. SG is an intelligent network that combines different entities actively involved in energy exchange [2]. The smart grid differs significantly when compared to the traditional grid. The first difference is that it enables bidirectional power flow. The Distributed Energy Resources (DERs) are able to make the transition to smart grid possible [3]. Actors that are capable to produce more energy from Renewable Energy Sources (RES) at a certain time should be able to sell it back to the market. The market itself has to evolve to a system that will get vast amounts of data as an input that need to be processed and by that create energy prices in the desired period. These data will be gathered from smart sensors and meters. It will also be transferred to demand response systems, whose task is to reduce peak demand. What is more, the SG needs to be resistant to cyberattacks. The ability to self-heal after power outages is also highly important. The transformation from the traditional grid to the smart grid will last a long time and the path of transition will require a challenging variety of technical, social, and economical innovations [4]. Microgrid (MG) is a concept of coordinated clusters of energy storage, sources, and loads that interact and cooperate with each other and the power system [5]. The purpose is to maximize the use of Renewable Energy Sources (RES). The social aspects 
of microgrids, such as increased awareness of climate catastrophe, job creation, and electrification of underdeveloped places, are also worthy of recognition. Numerous administrative actions to facilitate proper functioning and cooperation of MGs are implemented, e.g., tradable green certificates and feed-in tariffs [2]. There are further challenges associated with MG development, such as uncertainties of demand and supply using RES; design of appropriate Demand-Side Management (DSM) systems; and economical and reliable operation of MG with high penetration of RES, plug-and-play feature, voltage and frequency control mechanisms [4]. Due to the complexity of the MG, a flexible test system is an absolute need to ensure a reliable, secure and cost-effective evaluation environment.

MG can operate as an island or be connected to the grid or to other MGs. Moreover, multiple microgrids can also be interconnected to support and trade with each other, as described in [6]. Nowadays, in most countries, there is no possibility, or it is not easy, to implement for the MG to operate as an island. That trend will change in the following years. Working as an island means that MG has to produce enough energy by itself to power all the loads within this MG. The inverter unit can switch to islanding operation in one of two ways: unintentional and intentional [7]. The first instance, if it is not tested and handled properly, can pose a massive danger for utility workers, home appliances, or inverters. According to [8], if the root mean square (RMS) value per unit voltage will be out of range from 0.50 to 1.20 p.u. for at least $0.16 \mathrm{~s}$, or out of range from 0.88 to 1.10 p.u. for at least $2 \mathrm{~s}$, or the grid frequency deviation exceeds $\pm 1 \mathrm{~Hz}$, then DER should cease to energize and trip all units.

The inverters can combine their power and work together in parallel without the main grid. The possibility of parallel work of inverters in MG is a crucial aspect for the efficiency, flexibility, and reliability of the distributed generation system. Correct cooperation of inverter units serves to balance active and reactive power. That balance between real power $(\mathrm{P})$ and reactive power $(\mathrm{Q})$ must be maintained at an appropriate level. If voltage amplitude produced by one of the parallel inverters does not match, it creates reactive power circulations at the Point of Common Coupling (PCC). If additionally, the phase of the inverters is in mismatch, then both $\mathrm{Q}$ and $\mathrm{P}$ are circulating. Further, every inverter must produce voltage with an identical frequency. One of the hardware solutions to restrain circulating current is the transformer located at the output of the inverter [9]. It provides additional galvanic isolation and diminishes the DC composite of circulating current. The current produced by the inverters should be controlled and the circulating current has to be minimized. There are a lot of control methods, to tackle that issue. Methods are divided into two main categories: communication-based and methods based on droop control. A communication-based method like active current sharing or master-slave control is efficient for load sharing, but the necessity of high-speed communication infrastructure, as well as low redundancy, are the major drawbacks of this method [10]. Droop control does not require interconnection between inverters, although, in the most basic form, it suffers from the slow dynamic response and poor harmonic sharing [4]. Nevertheless, numerous improvements have been introduced to that method, e.g., angle droop control method, virtual impedance control method and adaptive voltage droop control method.

The smart grid emulator aims at allowing to model the behavior of the real grid. One of the main aspects is the distributed energy production in different scenarios. That is why we need a reliable energy generation block that is configurable and controllable. The inverter block we present and evaluate in this paper shall provide this functionality.

Nearly all the testbeds existing in the literature work with hazardous voltages. The paper presents a testbed where hazardous voltages can occur only in the primary substation and secondary substation emulation does not surpass $230 \mathrm{~V}$ AC. However, the input DC voltage of the inverter is equal to $35 \mathrm{~V}$ DC and the output voltage is $24 \mathrm{~V} \mathrm{AC}$. Besides the scaled-down voltages, the system is capable of mimicking grid behaviors with improved security measures. This approach made it possible to exclude concerns to making mistakes when studying the effects of individual elements influencing others. One of the key reasons to build a test bed is not to emulate only the normal work, but rather to test 
the emergency situations that might happened in electrical grid and the algorithms for adjusting to the disturbances or demand. The testbed with scaled-down voltages can provide these capabilities without creating extremely dangerous situations. This creates possibilities to implement more accessible testing environment. Furthermore, more risky scenarios like variety of grid faults can be simulated. Furthermore, care was taken to select relatively inexpensive components so that the overall cost of the emulator was significantly below the average MG emulators. What is more, the emulator uses a non-commercial inverter board, which has the advantage of being available to quickly exchange or replace given components like operational amplifiers, transistors, or filters. Moreover, during the design phase, the principle of using only readily available components was followed. Most of the MG testbeds require roomy laboratories in order to accommodate high-volume inverters that are larger due to, for example, larger inductors and radiators necessary in higher voltage applications. Another bulky and costly element is the grid simulator, which is also not needed in the presented design.

The primary objective of this paper is to investigate the validity of using the inverter that can serve as part of the MG emulator. This emulator requires a high degree of flexibility from the inverter, as the inverter itself can be used in many configurable options. Among these, the inverter should emulate a collection of several photovoltaic panels or a wind turbine, as well as small power plant. To meet these requirements, the inverter was put through a series of tests. Another novelty aspect is represented by the scaled-down voltages. It can be helpful to test risky scenarios without generating very hazardous conditions. Moreover, the tests presented in this paper can serve as a foundation for future multi-domain evaluation considering the energy management algorithms, communication (data exchange within the system) and simulation of energy market aspects.

The rest of this paper is organized in the following order. Section 2 covers the existing methods of microgrid testing. Advantages and drawbacks are discussed and the realized development has been described. In Section 3, the structure and the possible topologies were defined. Principles of the control system of the inverter as well as the communication with the master controller were described. Test scenarios and results are presented in Section 4. Finally, Section 5 provides concluding remarks.

\section{Testing in Microgrids}

Testing MGs can be done in a variety of ways, among others digital simulation, cosimulation, Real-Time simulation (RTS) Hardware-In-the-Loop (HIL), Controller-HardwareIn-the-Loop (CHIL), Power-Hardware-In-the-Loop (PHIL) and emulators. Several reviews and analyses have been made that compare these testing strategies and present a diversity of paths to create simulation within each category of test approaches [11,12]. To choose the desired strategy, the trade-off has to be made between the cost, test fidelity and test coverage [12].

The digital simulation consists of solving mathematical models with the use of highlevel language code and platforms. It is mostly used for the initial state of the system development. The benefits are flexibility, rapidity, versatility and no risk of damaging the equipment [13]. Cost varies depending on license period, but can reach several thousand EUR by year, in the case of choosing, the most popular solution, e.g., Matlab. Worth mentioning is that there are free simulation tools available, like Julia or GNU Octave. There is also a possibility that diverse power system modules can be simulated in different simulation tools; therefore, all simulations need to be merged in a co-simulation environment, for instance, mosaik.

A real-time system is performed on parallel processors. All parts of the system, for example, the control, protection and measurements, are modeled on the simulation platform [14]. The key issue of RTS is to ensure appropriate timescale, including idle time of processors [11].

When some parts of the system exist physically and the software part of the simulation is connected with the hardware, then the HIL approach is implemented. It is well known 
that HIL requires more time to build and investment costs are usually higher than in the previously mentioned approaches. A noticeable benefit is the behavior being closer to the real behavior of the given module. There are certain issues that ones designing the HIL system need to take into account, such as stability criteria and also time delay formed in simulation, which might be reflected in harmonics of output signal [13]. The CHIL systems add hardware to RT simulator, for instance, a power converter controller and signals are exchanged between them with the use of Analog-to-Digital or Digital-to-Analog converters [13]. That approach is widely used, and it gives scientists a valuable, small volume object to test basic algorithms like voltage control or power-sharing in MGs [15] or testing data exchange methods [16].

PHIL has a similar architecture to CHIL, but requires an additional power amplifier stage. This allows the use of devices that are involved in exchanging power such as solar inverters or generators. The appropriate selection of power amplifier requires a priori determination of bandwidth, expected accuracy and stability of the desired power amplifier [17]. Voltage and current amplitudes flowing through the PHIL system might be closer to the real values flowing in the power system, thus PHIL simulation has the ability to test more risky, complex and demanding scenarios. Examples of possible test cases are fault condition detection, protection validity or power exchange between units. A common approach to performing the test is to first simulate the system only in software and then compare the results in the PHIL environment. The authors of [18] present PHIL with the use of a commercial micro-inverter and put it through a series of stability tests. A complex test class was presented in [19], among others, abnormal voltage and frequency conditions, voltage regulation or unintentional islanding were investigated. Another paper [20] presents a simulation of a PV inverter, for voltage regulation in distribution networks, with the use of a variable voltage source. The system features and protection, together with reactive power behavior in case of fluctuating power capability of the PV array, were well tested. There are numerous PHIL simulators with the use of inverters. However, most of them use the commercial inverters that are dealing with dangerous voltages and are hard to configure.

The idea of the MG emulator is to imitate, as much as possible, the real behavior of the MG. Performing test scenarios on an emulator, instead of the real power system, has several advantages. For instance, there is no possibility of damaging the real equipment, it has the possibility of concentration of long tests in a shorter period by changing the timescale, it consumes less space, costs less and is more flexible [21]. One of the examples of such an emulator is presented in [5]. The authors present the Generator Emulator Controls that was designed to reproduce favorable grid-forming characteristic of a synchronous generator. The paper presents tests of plug-n-play ability in MG with 15-inverters. The testbed used a commercial inverter in the closed case, and it is slightly limiting the flexibility, although the authors sized the appropriate filter. In [22], the authors introduced an emulator with a voltage source inverter. The size of the test system is relatively small and due to the modular concept of the system, it is flexible to made changes in topology, but no test was performed to present that feature. In the paper, the authors test parallel operation as well as response to load step changes, but only for two values of the load and without measuring the frequency deviations at PCC.

\section{Structure of the Proposed Testbed}

\subsection{Structure and Possible Models to Create}

The testbed is a smart grid or an MG Emulator, in which the generation is based on the inverter module that is presented in this paper. The testbed emulates the power grid, but also includes a (wireless) sensor network that monitors the power grid. The main task of the emulator is to mimic a full-scale smart power grid to test and evaluate power management algorithms. To allow creating different topologies of the grid, it follows the modular approach. There are currently four classes of blocks that differ in functionality and can be connected to form the desired topology. These blocks are connected physically 
to define the energy flows, but they are also connected logically to define the hierarchical relations of the monitoring and control components available in each block.

The monitoring and control module available in each emulator block can be considered as a smart meter with sensing and control features. In a given emulator deployment, these devices are configured to be organized in a wireless sensor network. It is a kind of distributed brain of the whole emulator. Each smart meter consists of three components. It is based on the IHPNode $[23,24]$ platform with the main part-the FWNode board based on the MSP430F5438A microcontroller. This board is responsible for exchanging measurements and control data between the different emulator blocks. The second component is the ADCBoard - an extension board for the FWNode, whose task is to measure electrical quantities for three independent channels, using its 24-bit ADC converter. The measured electrical quantities are the active power, frequency, reactive power, apparent power, current, voltage, and the power factor. The last component is an adapter for the ADCBoard-the ACFrontEnd board. Its task is to adapt the voltage and current values in each channel to levels that are measurable by the ADCBoard.

Some of the blocks are further equipped with a management unit-a computing device with more power than a sensor network node, but it interacts with the emulator using these nodes and it runs the energy management algorithms. The management units exchange data between each other using the data exchange middleware [25] that also allows implementing distributed energy management algorithms according to the framework defined by the European e-balance project [26].

Primary Substation (PS) is the first block that is a part of the emulator. It is an equivalent of a large high voltage electrical substation in the full-scale power grid, whose task is to reduce the voltage level from high voltage (HV) to medium voltage (MV) and to distribute the energy to the underlying secondary substations. In the emulator the primary substation voltage ratio is $230 \mathrm{~V}(\mathrm{HV})$ to $48 \mathrm{~V}(\mathrm{MV})$. It is equipped with both, the smart meter module that monitors the voltage and current parameters on the MV side of the transformer, and the management unit.

The Secondary Substation (SS) is the second block in the emulator. Its task is to further reduce the voltage level from the MV to low voltage (LV) and to distribute the electrical energy of the area that is connected to it. The LV level in the emulator is defined at $24 \mathrm{~V}$. It is also equipped with both the smart meter module that monitors the voltage and current parameters on both sides of the transformer (MV and LV), and the management unit.

The emulator blocks can be connected directly to create the energy flows between these, but it is also possible to use the Transmission Line (TL) blocks for that. Similar to full-scale transmission lines, the main purpose of this block is to transmit electrical energy. The transmission line block has a specific structure that allows to split one input into three independent outputs or to accumulate up to three independent inputs into one output. The smart meter module monitors the voltage and current parameters and controls switches that enable cutting of each of the three outputs/inputs. Furthermore, the transmission line block has the capability to emulate electricity transmission losses/influences by changing its parameters using passive electronic components between input and output.

The TL block was primarily designed to test energy management algorithms, such as the reaction to (emulated) transmission line faults. It can thus be used to detect blackouts and rearrange the topology to distribute power from another source. A TL block is not equipped with a management unit and is thus controlled by its respective parent substation. The link between the TL and the substation is logical on the wireless sensor network level, connecting the smart meter modules. It gives the opportunity to measure electrical quantities for the entire sub-grid, but also just for a selected branch of the sub-grid. The parent substation has also the possibility of cutting off the selected sub-grids in the whole grid using commands that are dedicated for this purpose.

The last and the most complex block in the emulator is the Prosumer block. This block is responsible for the emulation of power consumption and production on the LV or MV level. This is the block that contains the inverter presented in this paper. It can 
emulate a single household, either only consuming energy, but also one with, for instance, a photovoltaic system. However, it can also emulate a larger energy producing site, like an independent solar or wind power plant. On the energy grid emulation level, the prosumer block consists of the inverter module and the energy-consuming module connected in parallel. Both current flows are monitored using the smart meter module independently. This allows to emulate scenarios relevant for different regulatory settings in different countries, i.e., the energy consumption and production can be regarded individually or as a sum. The prosumer block also includes the management unit to run energy management algorithms.

All these block classes of the emulator provide the opportunity to flexibly design multiple topologies and test many smart grid approaches on them. An example topology to be emulated is presented in Figure 1.

LV grid

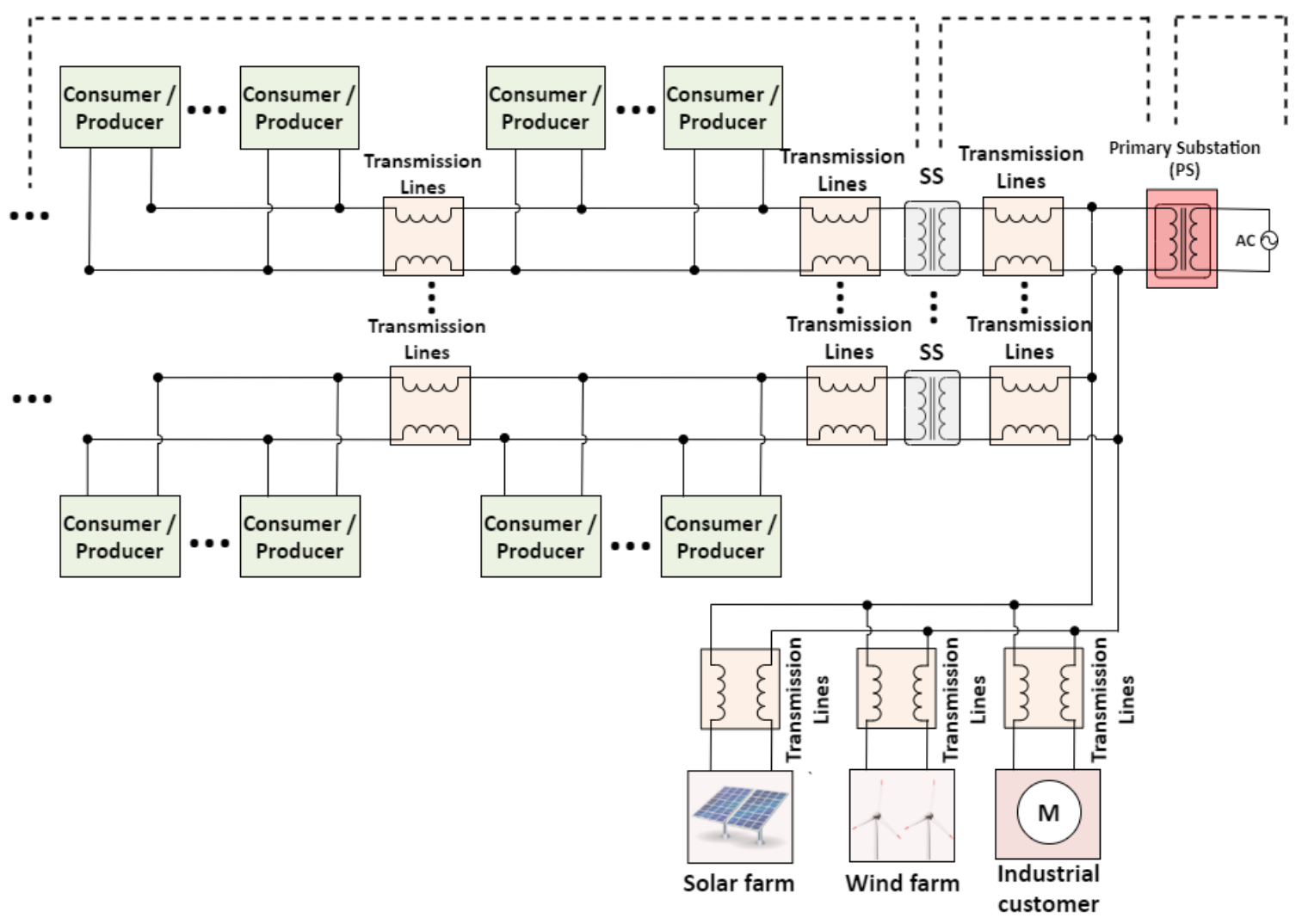

Figure 1. An example energy grid topology.

The main issue within the emulator related to the inverter module is its configurability and control to prevent hazardous situations. The energy generation in different prosumer blocks is summarized, i.e., the outputs of inverters are connected almost in parallel, thus a proper definition of the inverter behavior and reliable control mechanisms to realize that behavior are a must.

\subsection{Principles of Operation and Control}

The detailed software and hardware architecture is described in master thesis [27]. It provides a comprehensive explanation of the control mechanism as well as the hardware design procedure. Nevertheless, the principles of operation and control are presented in this section. To control voltage and current flow within the emulator, two loops with proportional-integral (PI) controllers were used. The current control loop is an inner loop. The operation of this loop starts with the measurements of the instantaneous values of currents in both leg branches of the inverter bridge. That is performed via the current 
sensing circuit that measures current by calculating the drop on the shunt resistor at the low side of the inverter branch. Then, the voltage drop is transmitted to the integrated circuit with an operating amplifier, whose task is to process the signal to the appropriate level. Then, the signal is transmitted to the Analog-to-Digital Converter (ADC), where the control unit of this device will process the input signals and generate PWM signals. By manipulating the PI coefficients, it is possible to shape the characteristics of the PWM signals. By changing the shunt resistor's values or components included in the current sense circuit, it is possible to adjust the resolution and accuracy of the measurements. Hardware components are easily accessible for replacement, and software coefficients can be changed via SPI, as described in Section 3.3. The outer voltage control loop senses the voltage at the output of the inverter and compares it to a desired voltage reference, that is $24 \mathrm{~V}$. The measurement circuits are shown in Figure 2.

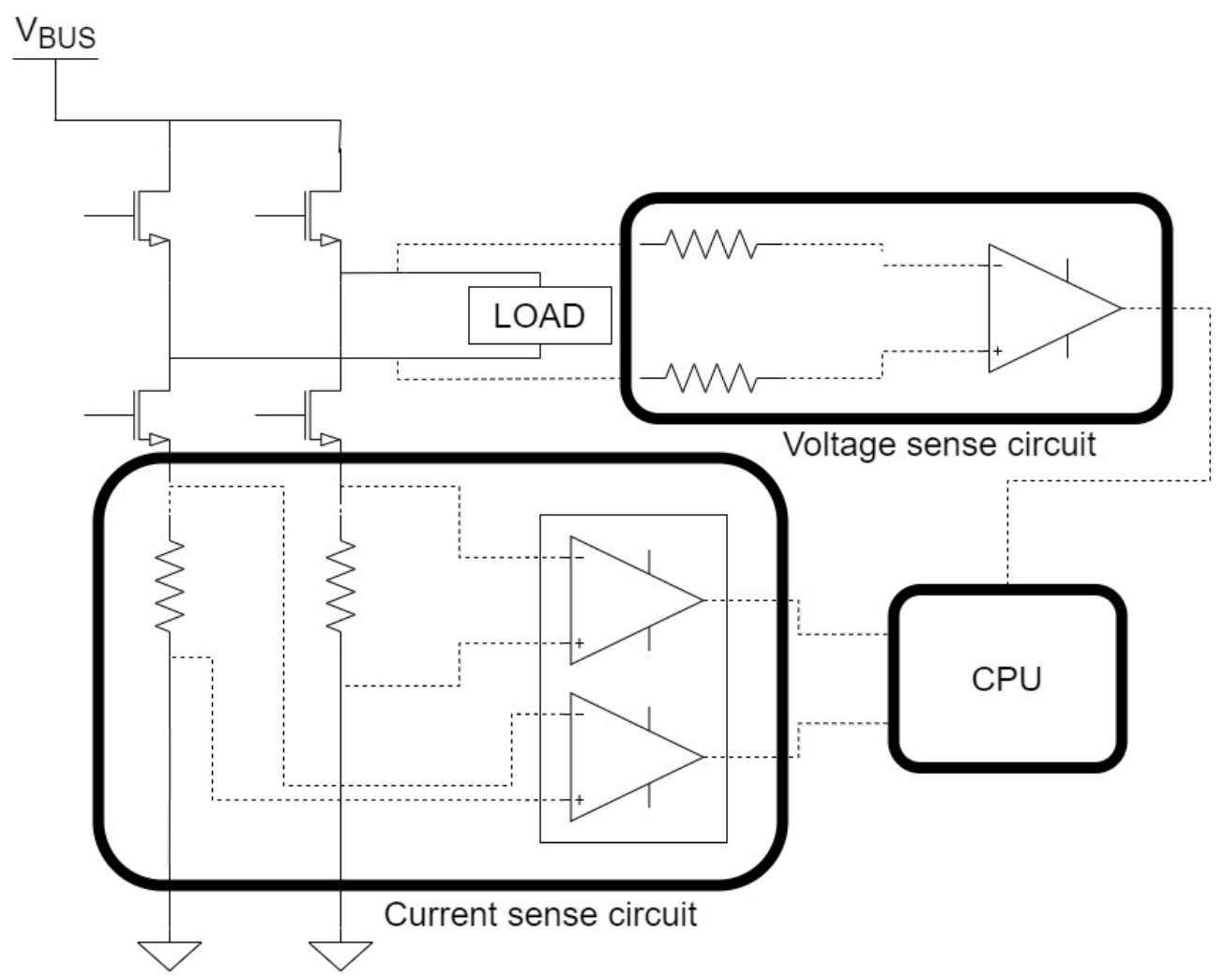

Figure 2. Current and voltage sensing loops.

To work in parallel with the grid, or with other inverter units, the synchronization of phase has to be accomplished. The most widely used, and the most researched method to ensure synchronizing is the implementation of the phase-locked loop (PLL). This structure is based on a feedback loop, where a measured output voltage signal is subtracted from a reference signal, which is the grid voltage. The result of the subtraction is an error signal. The error signal is minimized by an internal discrete low-pass filter. The discrete oscillators finally lock the generated output signal with the phase angle of the input signal. The lock of the signal is proportional to the performance of this system. The structure of this system contains three basic functional blocks:

- Phase Detector (PD)—the main purpose of this block is to compare the input sinusoidal signal with the locked sinusoidal signal and to produce error signal out of it.

- Loop filter (LF) - it is a low-pass filter that clears the error signal by damping output noises and high frequencies from the signal.

- Voltage Controlled Oscillator (VCO) — this block produces the output signal as well as the locked signal with respect to the nominal frequency. 
Unfortunately, the grid frequency can be close to the cut-off frequency of the PhaseLocked Loop. Therefore, additional blocks in the PLL system are required. Some approaches to improve PLL structures are using Adaptive filtering or Second-Order Generalized Integrator (SOGI) [28]. In both of the methods, the loop filter transfer function must be implemented and the coefficients of that filter have to be calculated with the discretization previously performed. The values have to be carefully calculated and tuned. In the project presented in this paper, the SOGI method was chosen.

When the inverters are working in parallel, one of them, namely, the grid-forming inverter, must set the reference signals for the rest of the inverters [29]. The other inverters are following the reference and synchronize to the grid-forming unit. In case of grid synchronization work, all inverters are grid-following units. When the intentional or unintentional islanding occurs, then one of the inverters must be transformed into the grid-forming inverter. This case is covered in the tests. If multiple inverters work in parallel and the grid-forming inverter fails, another inverter must take its role to ensure the grid stability.

The activity of the inverter depends on its current state. The state machine is presented in Figure 3. At first, the inverter is in state 0 , where it is inactive. The inverter is waiting for the command to start operation. If the incoming command arrives, then the inverter switches to state 1 . There, the inverter checks, through the DC voltage sensing circuit, if there is a DC voltage higher than $34 \mathrm{~V} \mathrm{DC}$. If that condition is met, it changes to state 2 , and then, the inverter is sensing the output voltage. In this state, depending on the presence of the grid voltage, the inverter can go into the synchronization or the islanded mode. First, if the grid voltage is present, it means that the main grid or another inverters already power the load. In that case, the inverter control unit needs to make sure that the inverter is capable to produce enough current according to references that are set to PI controllers. If this requirement is met, then the transistors are switched on, and the inverter starts to work in parallel with the other inverter units, on the grid. It remains in that state, until it will not receive a command from SPI to turn off, or until any of the protections trigger. If the grid voltage is not present, then it simply goes to islanded mode and powers the loads by itself. The rules for returning to state 0 are the same as for synchronous operation.

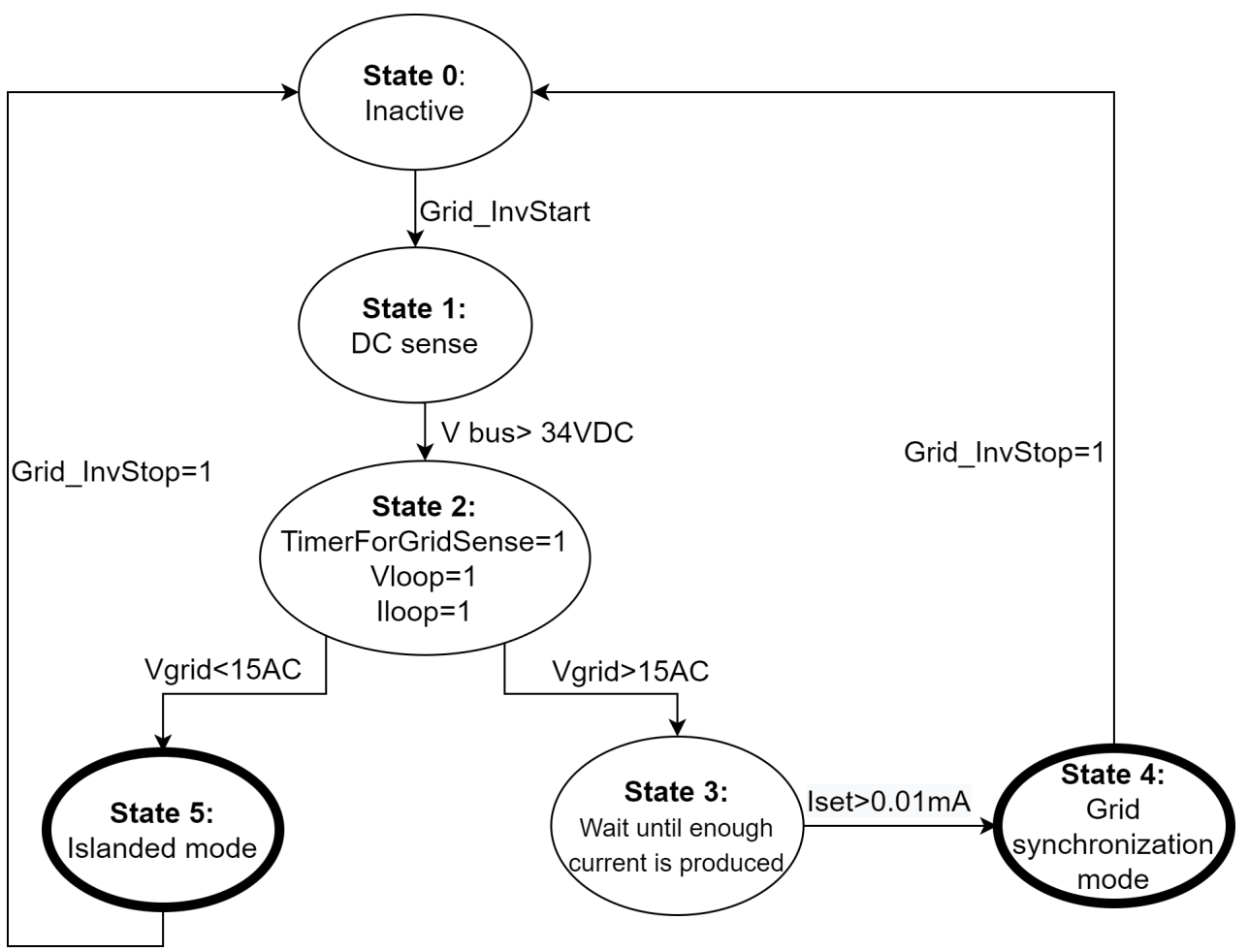

Figure 3. State machine of inverter. 


\subsection{Communication between the Inverter and the Master Controller}

The communication is done by means of SPI. The SPI is configured with the guidance from in [30]. The SPI is configured to send a bitstream of length equal to 16 bits. The inverter unit is always a slave. The four-wire mode is chosen, that is Slave-Output-Master-Input (SOMI), Slave-Input-Master-Output (SIMO), Slave-Transmit-Enable (STE) and SerialClock (CLK). Data are transmitted at a baud rate of $156.25 \mathrm{KBps}$. The transmission of data is performed with the use of the First In, First Out (FIFO) buffer. Each member of the conversation, namely the transmitter and the receiver, is equipped with the shifted register and the buffer. In the beginning, the master initializes communication, by setting the clock. When the data are written to the buffer register, the start of the conversation is performed. Then, the data are sent, bit by bit, on each rising edge of the clock from the shifted register. The data are saved on the falling edge of the clock signal. The data from the Most Significant Bit is transmitted by pin SPISIMO, and the receiver is collecting the data from the SPISIMO pin. On the receiving side, initially, the data is assigned into the shifted register, after sending the required numbers of bits, data is passed from shifted register to buffer. After that operation, the appropriate flag is set and then data is ready to be read. Afterwards, the interrupt flag is being set, and if the master does not have anything more to transmit the clock is stopped.

In the proposed MG emulator, the management unit in the prosumer block controls the inverter in that block. The smart meter module is the interfacing in that case. It is the master and the inverter is the slave. When the master wants to control the inverter, the information is transmitted using a 16-bit hexadecimal value that is $0 \times A B B B$, where: $A$ is the ID of the parameter and $B B B$ is the value of the parameter. For instance, the variable that turns on or off the inverter has an ID equal to 1 . Setting logical 1 to that variable turns on the inverter. Therefore, in order to turn on the inverter, the master controller has to write a command 0x1001 via SPI. The slave will receive data, encode it, and then change the parameters.

Further possible commands are:

1. Setting power reference. The inverter can control the current it is producing. When the current is lowered, then other units should compensate for the voltage drop and increase current as investigated in Section 4.4.

2. Prioritizing of the inverter. There are 3 levels of priority: low, medium and high. By default, all units are configured at medium level. When priority is set to high, then the inverter will try to produce more current, and similarly setting priority to low will decrease the current.

3. Allowable power limit. The upper and lower ranges of producing power can also be controlled. If the upper limit is set, and a given inverter is capable of producing more power, then it will lower the current, to meet the upper boundary. In case the inverter is not capable of producing more power, than the lower limit indicates, then the inverter is turned off.

4. Possibility of islanded mode. Master unit can ask for possibility of transition into the islanded mode. After checking the parameters, the inverter gives an answer about the possible transition.

5. Request the state. The inverter navigates through the state machine as described in Section 3.2. After receiving the request, the inverter unit responds in which of the state it currently operates, among: (i) powered, not active, (ii) islanded mode, (iii) synchronization mode as a master that is grid-forming control, (iv) synchronization mode as a slave that is grid-following control.

6. Request the measurement. The microcontroller is equipped with the ADC, thus it measures electrical quantities within the inverter board like the output AC voltage, branch current, input DC voltage, frequency. These values can be requested and send in predefined intervals. 


\subsection{Testing Circuit}

To test the behavior of microgrids with different number of inverters with different configurations, several test scenarios were defined. In order to simplify the test procedure, test schemes were defined using the inverters only.

Figure 4 presents the default circuit for all testing scenarios unless otherwise stated. In the preface of every test scenario in Section 4 , the number of inverters $n$ participating in the test scenario as well as the preset connection of relays SWn1 and SWn2 are defined. The physical test circuit is presented in Figure 5.

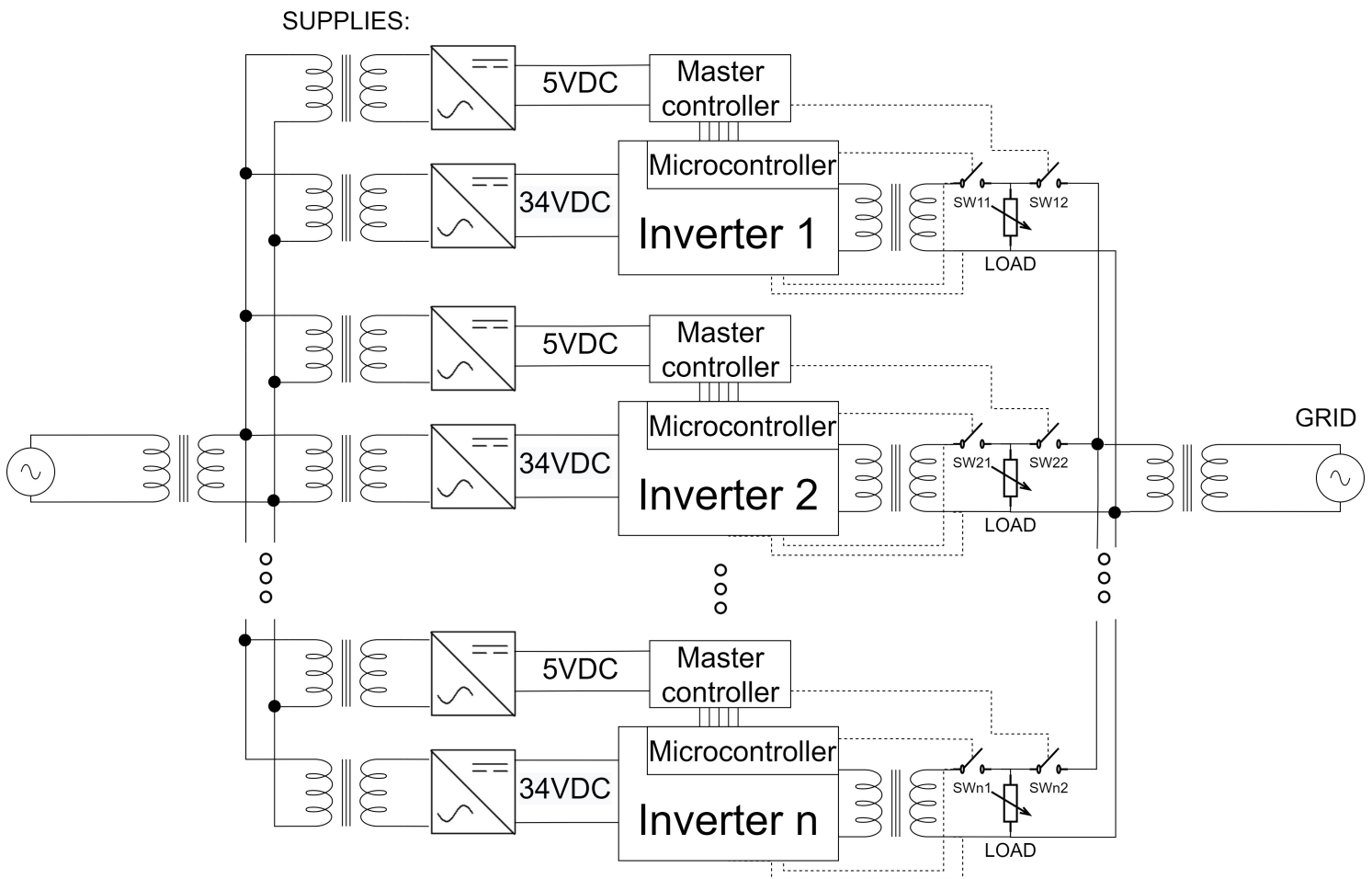

Figure 4. Block diagram of testing circuit.

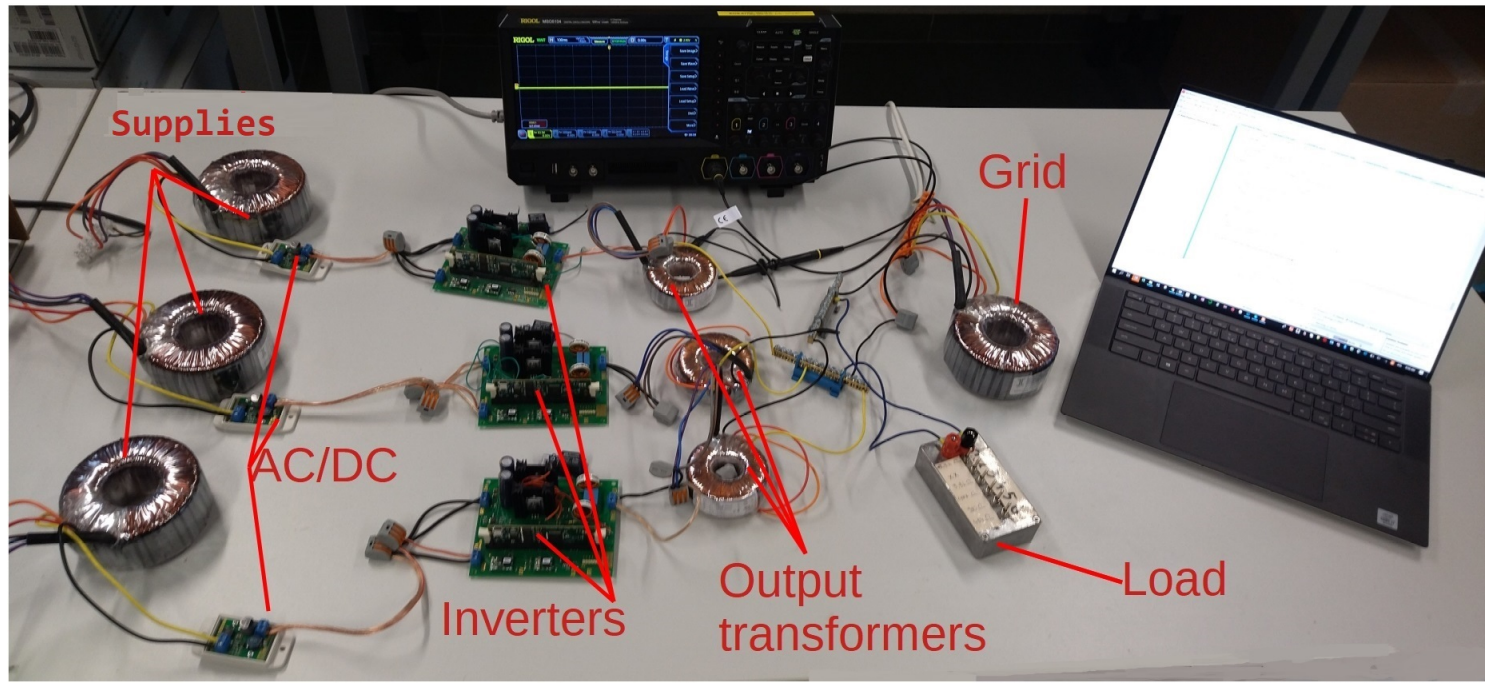

Figure 5. Physical testing circuit.

\subsection{Measurements}

The voltage and current measurement samples are captured by the RIGOL MSO5104 oscilloscope. The data are saved and eventually processed for RMS values or frequency. 
The moving RMS values are computed in a window of five cycles of the $50 \mathrm{~Hz}$ sinusoidal waveform that is recommended by the standards for transient measurements [8]. The sample rate of oscilloscope measurement varies between test cases. In load transient changes, the sample rate was equal $500 \mathrm{kSa} / \mathrm{s}$, in connection and removal, the sample rate was within range (depending upon sub-case) from $50 \mathrm{kSa} / \mathrm{s}$ to $5 \mathrm{kSa} / \mathrm{s}$. The voltage is measured directly at PCC and currents are measured at the output of the transformer via a current sensor circuit that contains a shunt resistor and Current-Sense Amplifier INA240 [31].

\section{Test Scenarios and Results}

This section presents the realized test scenarios and obtained results.

\subsection{Connection and Removal of Inverters in Parallel}

To assure efficient operation of inverters, the droop control is used. The main principle of a droop control method is that the voltage amplitude and the phase follow the reference when droop of load current changes. Active and reactive power flowing through parallel inverter system can be expressed as on Equations (1) and (2), respectively.

$$
\begin{gathered}
P=\frac{E V}{X} \sin \phi \\
Q=\frac{E V \cos \phi-V^{2}}{X}
\end{gathered}
$$

where $X$ is the output impedance of the inverter, $\phi$ is the phase angle, $E$ is the amplitude of output voltage produced by the inverter and $V$ is the load voltage.

Therefore, $P$ is mainly dependent on $\phi$, while $Q$ depends on $E$. Accordingly, the voltage droop is introduced as Equation (3) and the phase drop as Equation (4), on which $E^{*}$ and $f^{*}$ are the open circuit equivalents, and $m$ and $n$ are the droop coefficients. $P_{0 i}$ and $Q_{0 i}$ are ratings of $\mathrm{P}$ and $\mathrm{Q}$, while $P_{i}$ and $Q_{i}$ are real-time values $\mathrm{P}$ and $\mathrm{Q}$.

$$
\begin{gathered}
E=E^{*}-n\left(Q_{0 i}-Q_{i}\right) \\
f=f^{*}-m\left(P_{0 i}-P_{i}\right)
\end{gathered}
$$

The ability of parallel work of inverters is one of the key aspects of MGs. The presented paper includes a test with up to $n=6$ inverter units working in parallel. The inverters work without the main grid that is, switches $S W n 3$ were disconnected, $S W n 1$ and $S W n 2$ were connected. The distortion of sinusoidal waveform of output voltage in the case of six inverters working in parallel does not differ significantly from the single inverter operation as presented in Figure 6. A slight decrease in RMS voltage may be observed. Nevertheless, the value is not meaningful and the capacity of the grid in that configuration is not weakened and, there is still possibility to attach other units.

The test case is divided into three subcases. The first one involves the connection of six inverters sequentially, and then the removal of them in the same manner. The total time of the test takes $200 \mathrm{~s}$. Each new inverter was connected every $20 \mathrm{~s}$. That time interval allows to equalize the voltage and move the system back to steady state. The second subcase was testing the connection and further removal of two inverters at the same time. It allows to test a possibility of connection more than one unit at the same time if the system urgently requires lots of energy. What is more, it allows to analyze the merge of two unconnected MGs. That can happen when one MG cannot produce enough energy by itself to satisfy the demand and other MG that can produce a surplus of the energy can fill the energy gap. The removal can symbolize the fault of the given unit, group of inverters, or transmission line fault. The third subcase, as the previous one, checks the possibility to connect multiple units. In this case to a MG with two inverters the connection and removal of another four inverters at the same time was analyzed. The results are presented in figures, where the top 
subplot presents the voltage RMS per unit value, the middle subplot presents the number of inverters working in parallel, and on the bottom subplot, the frequency is presented. Note, that for a given subcase there might be different ranges on the y-axes of the plot and also every subcase takes a different time.

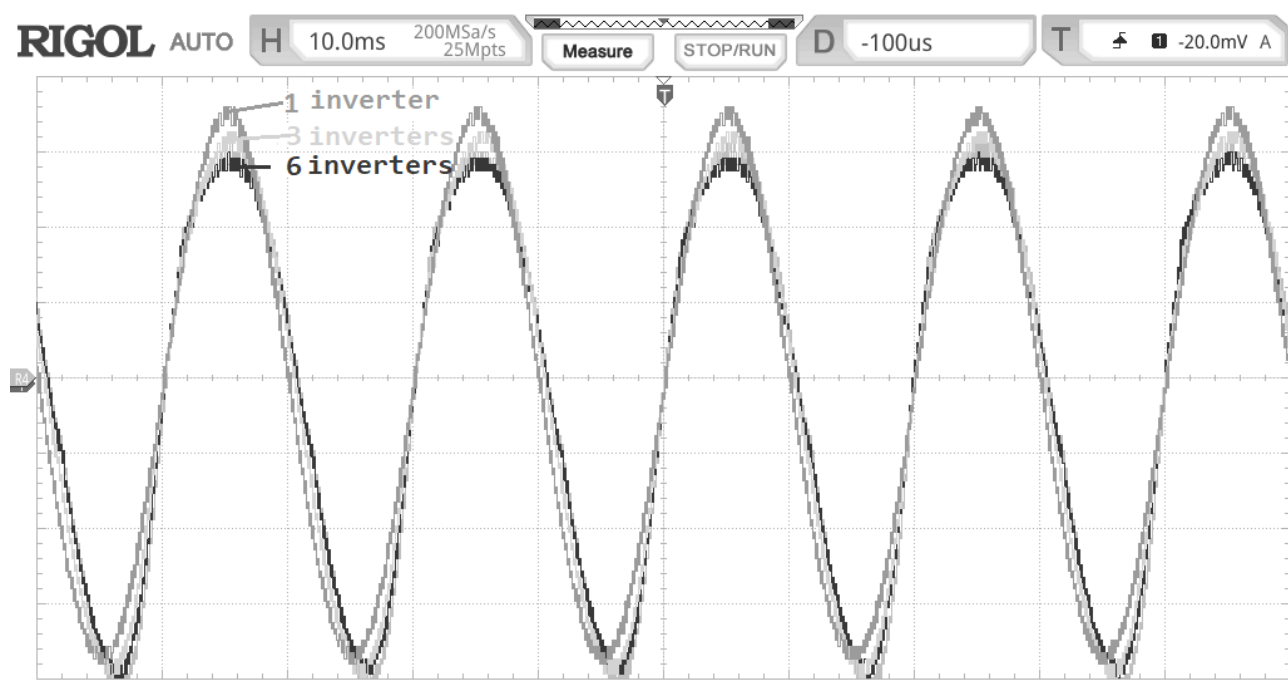

Figure 6. Comparison of the output voltage of 1,3 and 6 inverters working in parallel.

The guideline of allowable voltage levels and time of exceeding these levels are taken from IEEE standards [8]. These limits are presented in Figure 7. After connection or removal, the voltage should not exceed boundaries from 0.50 to 1.20 voltage (p.u.) in a period of maximum $0.16 \mathrm{~s}$.

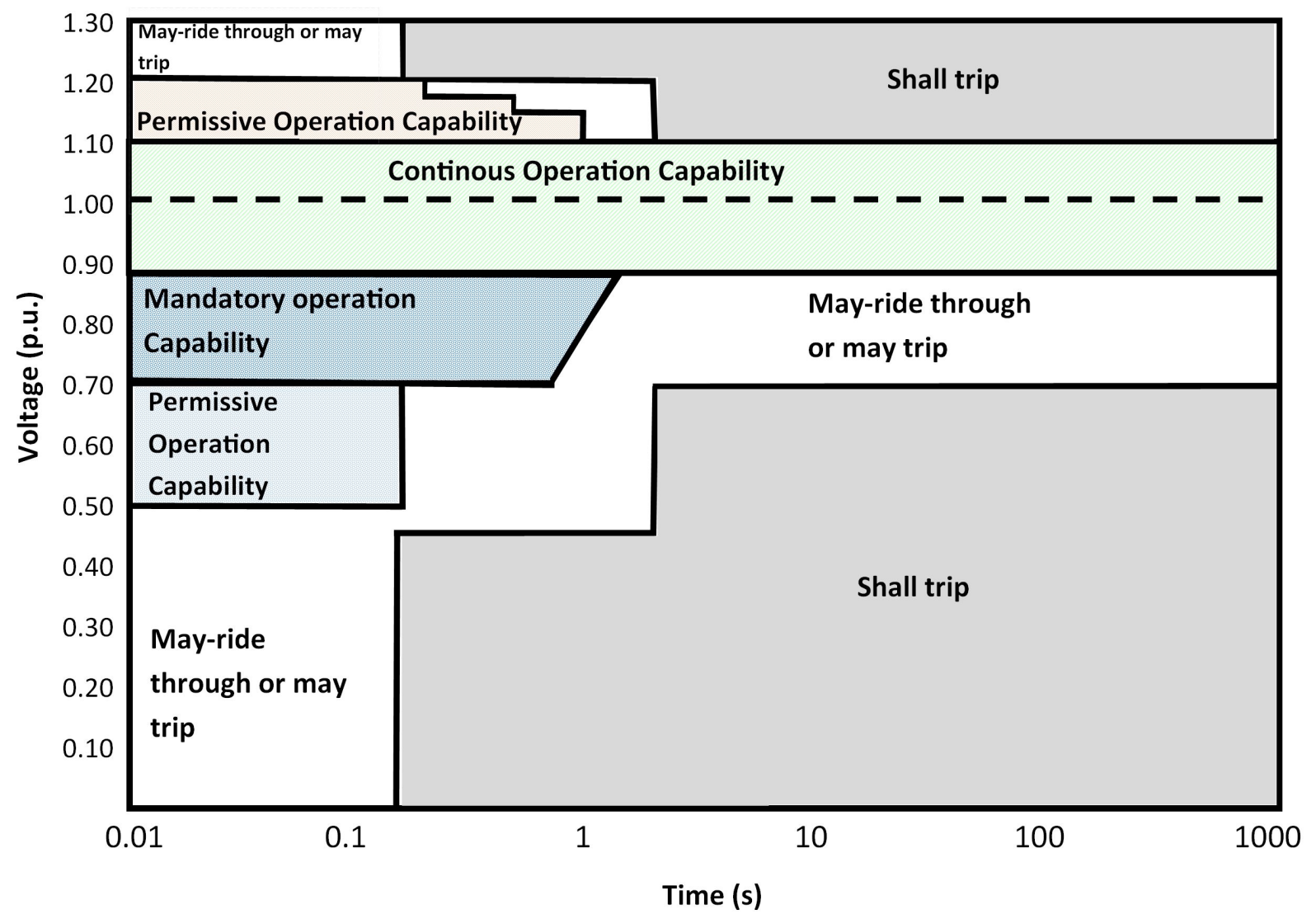

Figure 7. Allowed voltage levels, adapted from [8]. 
The first sub-case results are presented in Figure 8. From the results, it can be concluded that the voltage spikes have the biggest amplitudes on the connection and removal of the second and third inverter. Then, the inverters working together, starting to resemble a stronger grid. Each subsequent inverter is loaded with a smaller value of current, and the voltage spikes can be absorbed by higher numbers of output filters. The acceptable limits are not violated in any connection or removal. Each change of inverter numbers creates frequency oscillations. In every case, the period of oscillations never exceeds $0.20 \mathrm{~s}$. The frequency spikes were significantly higher when a new inverter was connected than removed. The removal of the inverter results in smaller voltage spikes, and that is the reason why the connection is riskier for the system. The moment of connection and removal of the same inverter is presented in Figure 9. After the removal, the inverter comes back to steady-state longer than in the case of connection.

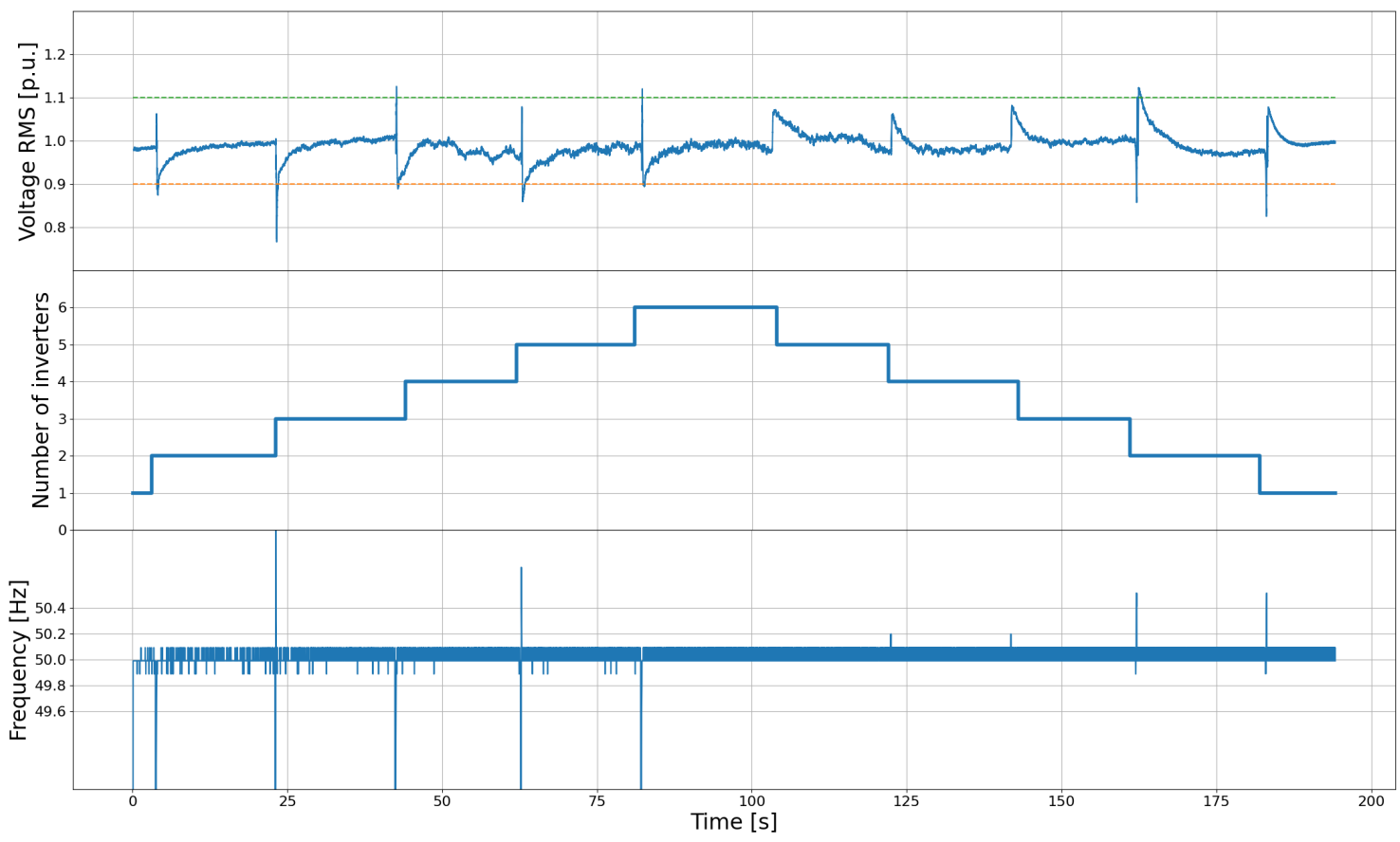

Figure 8. The sequence of connection and removal following inverters.

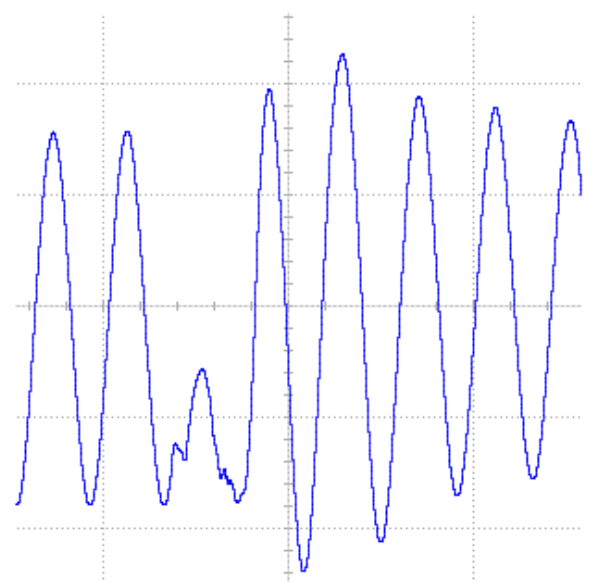

(a)

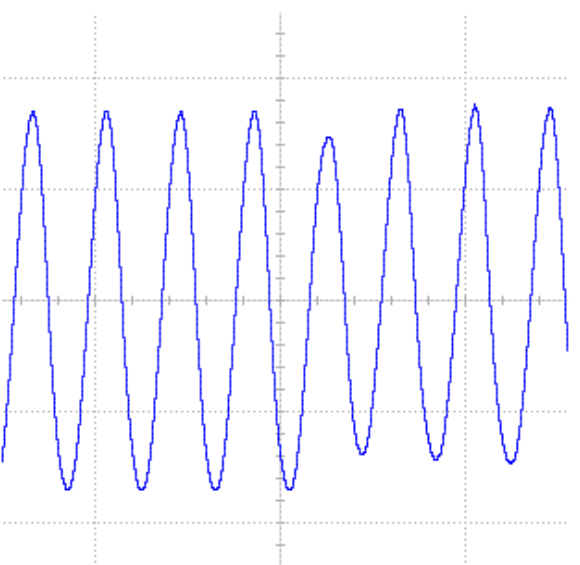

(b)

Figure 9. Connection (a) and removal (b) of the inverter. 
The second sub-case presents the connection and removal of two inverters. The voltage spikes during the event of connection sometimes exceed the upper level and reach a value of approximately 1.45 volts per unit as presented in Figure 10. The tests were repeated multiple times and in some cases, the first group of two inverters and in other repetition, the next group of inverters generates bigger spikes. The random nature of the voltage spike amplitude depends upon the phase of voltage at PCC at the time of connection. If the phase difference is further away from 0 degrees, then the connection might be more difficult and creates more spikes. The spike lasts less than $0.1 \mathrm{~s}$, thus it falls in "may ride-through or may trip" zone.

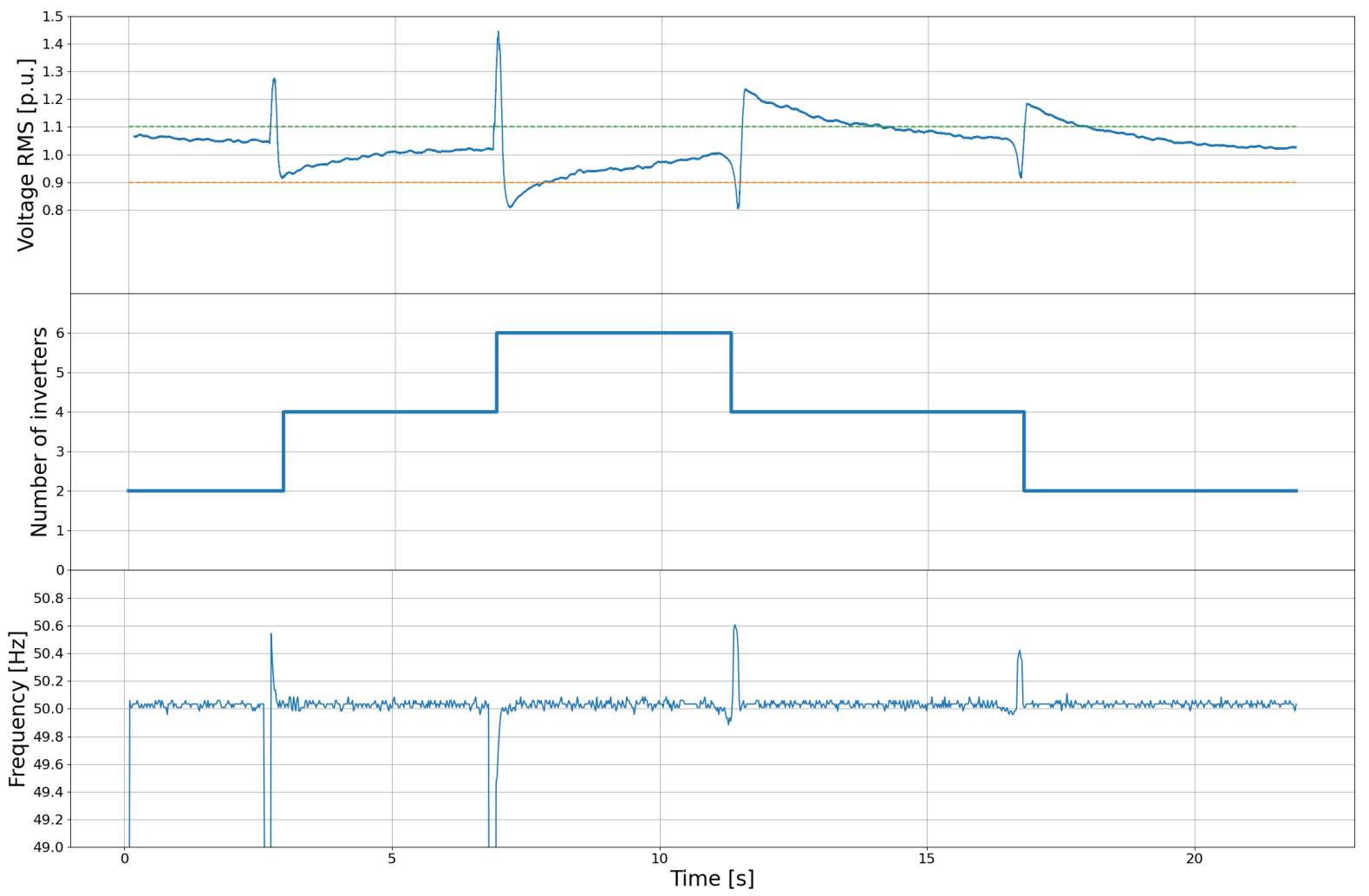

Figure 10. The sequence of connection and removal, two inverters at the same time.

The connection and removal of 4 inverters into 2 inverters MG has violated levels defined in standards (see Figure 11). In that sub-case, the emulator fails most of the repetition. If the connection of MGs with that much indifferent power level is needed, then voltage spikes protection devices alike varistor, surge protectors, or special protection circuits are necessary to implement. 


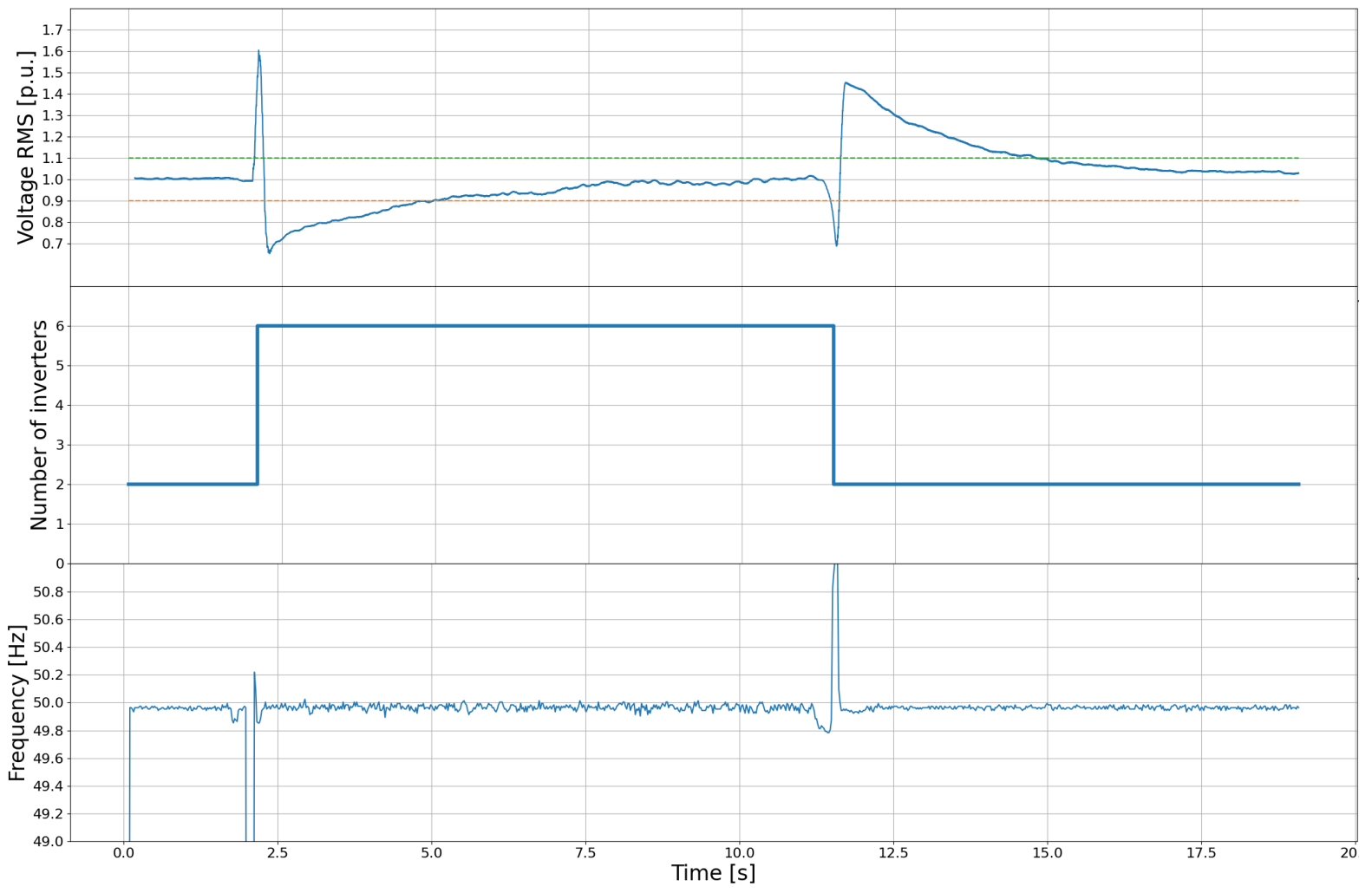

Figure 11. Connection and removal of neighbor MG with twice the power of the first MG.

\subsection{Adaptation to Load Changes}

The circuit used for testing is shown in Figure 4 for $n=6$ inverters. The inverters work without the main grid, that is, switches $S W n 3$ were disconnected, and $S W n 1$ and $S W n 2$ were connected. The purpose of this test was to verify that the voltage would stabilize during the load changes and the possible overshoots or undershoots of load changes should not exceed standards presented in Section 4.1. The load was switched between $250 \Omega$ to $16 \mathrm{k} \Omega$. With nominal RMS voltage equal to $24 \mathrm{~V}$, the following load values were used: $2.30 \mathrm{~W}, 1.15 \mathrm{~W}, 0.60 \mathrm{~W}, 0.30 \mathrm{~W}, 0.15 \mathrm{~W}, 0.05 \mathrm{~W}$. According to standards, the voltage should not exceed $\pm 10 \%$ of nominal value. Therefore, these levels are marked with green and orange dashed lines. The results are presented in Figure 12.

At the top subplot, the RMS voltage per unit is presented. The green dashed line stands for the upper allowable voltage limit and the yellow dashed line stands for the lower limit. Initially, six inverters start to work in parallel and achieve a steady state with a maximum load within $2 \mathrm{~s}$. The acceptable voltage and frequency ranges are presented in Figure 7. The series of events was triggered to change the load to smaller values. After that, maximum step change was done and load from $0.05 \mathrm{~W}$ was changed in $t=54 \mathrm{~s}$ to $2.30 \mathrm{~W}$ and then back to the minimum value. The particular times of switching the variable loads are presented in Table 1. First, the load from $2.30 \mathrm{~W}$ was changed to half of its value. The upper voltage was violated only for around 0.03 voltage p.u. The duration of exceeding boundaries was less than $1 \mathrm{~s}$, thus no protection devices should trip. Each subsequent change of the load has a smaller overshoot, and shorter settling time, until changes between maximal values. The heavy load changes may lead to huge voltage drop resulting in cascading inverter fault and finally to black-out [9]. In $t=54 \mathrm{~s}$, the change from minimal tested value to maximum tested value was performed. The undershoot of RMS voltage was equal to 0.11 p.u. below the lower boundary, and subsequent overshoot on change from maximal to minimal load was 0.16 p.u. above the upper level. The detailed results are presented in Table 1. The system frequency is resistant to load changes. During the testing, multiple test runs were performed in order to ensure the repeatability of this test setup. 


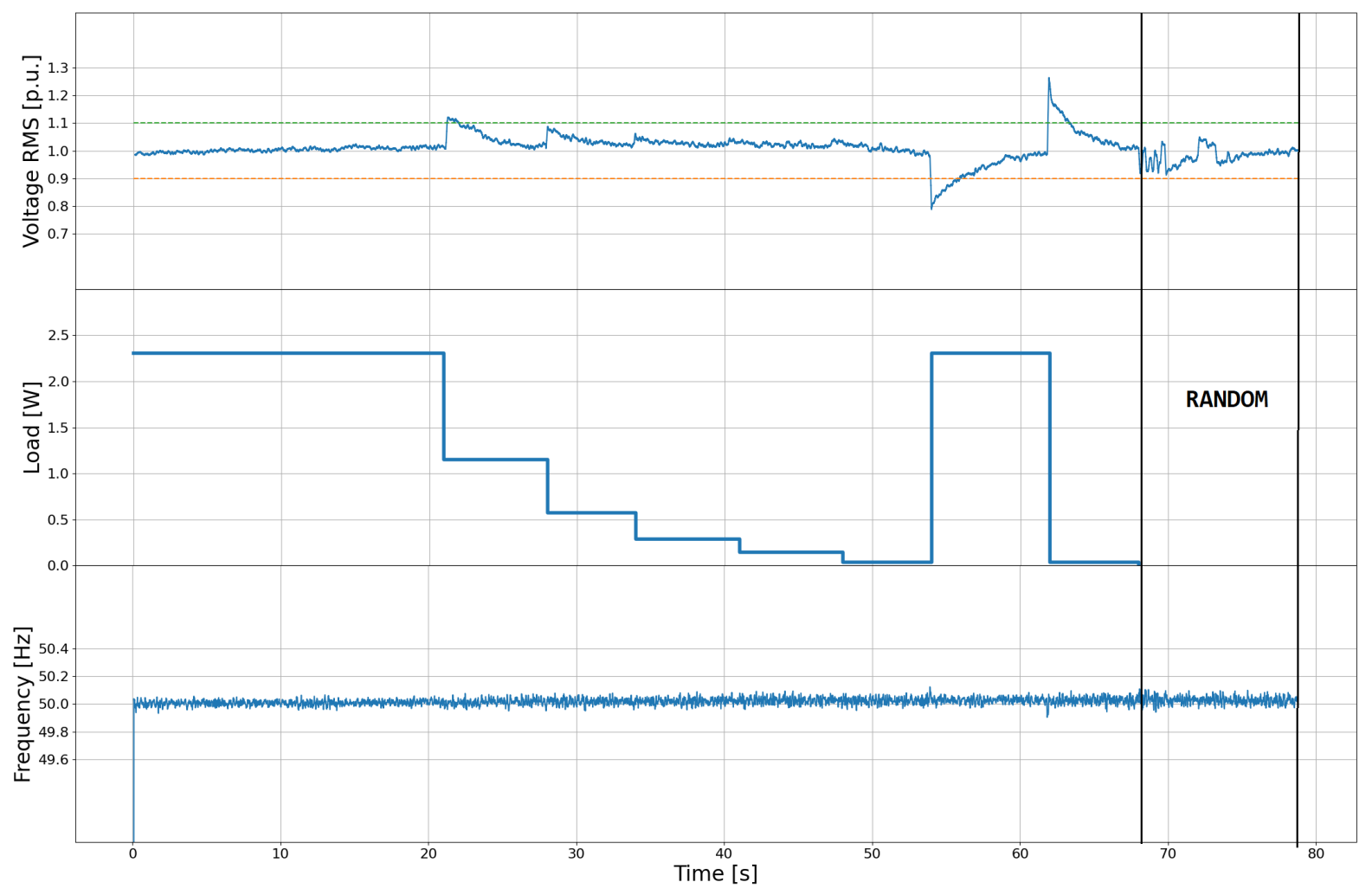

Figure 12. Electrical quantities of switching load test.

Table 1. Resistance values of variable load at the time.

\begin{tabular}{ccccc}
\hline Time [s] & Load [W] & $\begin{array}{c}\text { Settling Time } \\
\text { [s] }\end{array}$ & $\begin{array}{c}\text { Overshoot } \\
\text { [V p.u.] }\end{array}$ & $\begin{array}{c}\text { Undershoot } \\
\text { [V p.u.] }\end{array}$ \\
\hline $0-21$ & 2.30 & 2.6 & 0.11 & - \\
$21-28$ & 1.15 & 1.4 & 0.08 & - \\
$28-34$ & 0.60 & 0.6 & 0.06 & - \\
$34-41$ & 0.30 & 0.2 & 0.04 & - \\
$41-48$ & 0.15 & 0.1 & 0.03 & - \\
$48-54$ & 0.05 & 0.05 & 0.02 & 0.21 \\
$54-62$ & 2.30 & 7.2 & - & - \\
$62-68$ & 0.05 & 5.1 & 0.26 & 0.08 \\
$68-78$ & random & - & 0.05 & \\
\hline
\end{tabular}

Afterward, in $t=68 \mathrm{~s}$, when the system returns to a steady-state, the load started to change randomly in a dynamic way in a short interval of hundreds of milliseconds within ten seconds. That has caused only small voltage disturbances and the system responds quickly and boundaries of applicably voltage levels were not violated.

\subsection{Detection of the Anti-Islanding}

In the proposed emulator, a combination of two local, passive methods is used: Over/Undervoltage Protection and Over/Underfrequency Protection [32]. The standards are described in Section 1. The following test is designed to prove the appropriate operation of unintentional transition from the grid-connected mode to the islanded mode. The test circuit is presented in Figure 4 for $n=1$ and switches SW11 and SW12 are closed. The DUT is an inverter that is synchronized with the main grid. In steady-state operation, the grid fault was simulated via disconnection of the main grid from the inverter unit. In that occurrence, the flag on the inverter was raised that indicates that the grid voltage is below the applicable level. The task of the DUT is to transition from the grid-following mode to the grid-forming mode. 
The moment of transition is captured on the oscillogram and presented in Figure 13. The oscillogram shows that the inverter requires two periods that is $40 \mathrm{~ms}$ to change between the two previously mentioned modes. Note that during the transition, no voltage spikes occurred, thus there is no possibility to damage the Device Under Test (DUT) or any home appliances. The transition was repeated multiple times in order to consolidate results. In Figure 13, the transition is made instantaneous. Generally, a delay is worth implementing once the occurrence of under voltage has been detected. This is relevant to not force the islanded operation in case of temporal voltage disturbances. That ability is also present in the emulator, and the delay can be freely adjusted to current standards. For the test, $50 \mathrm{~ms}$ delay was selected. To delay duration, a constant of $40 \mathrm{~ms}$ should be added, for the time required for transition. The result is presented in Figure 14.

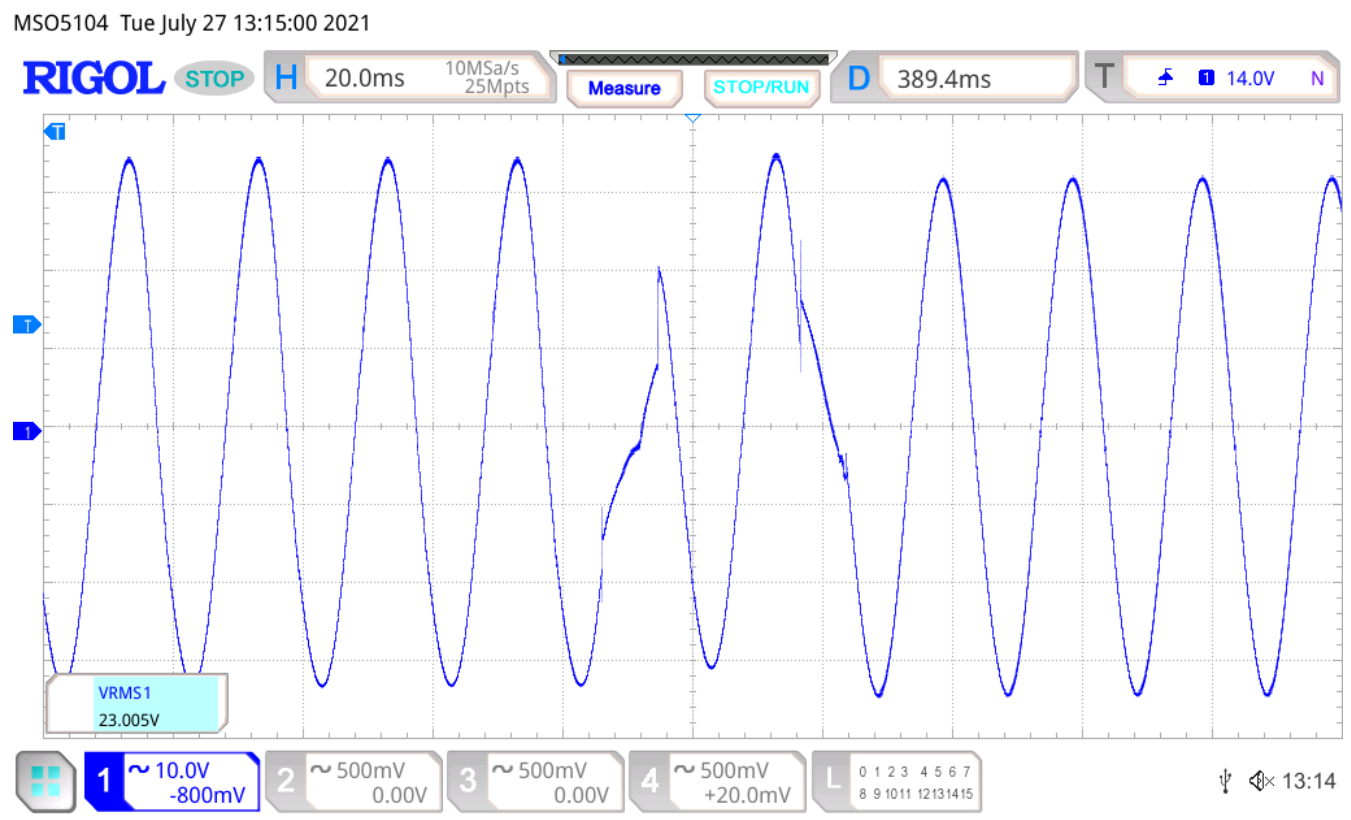

Figure 13. The transition from grid synchronization mode to islanded mode.

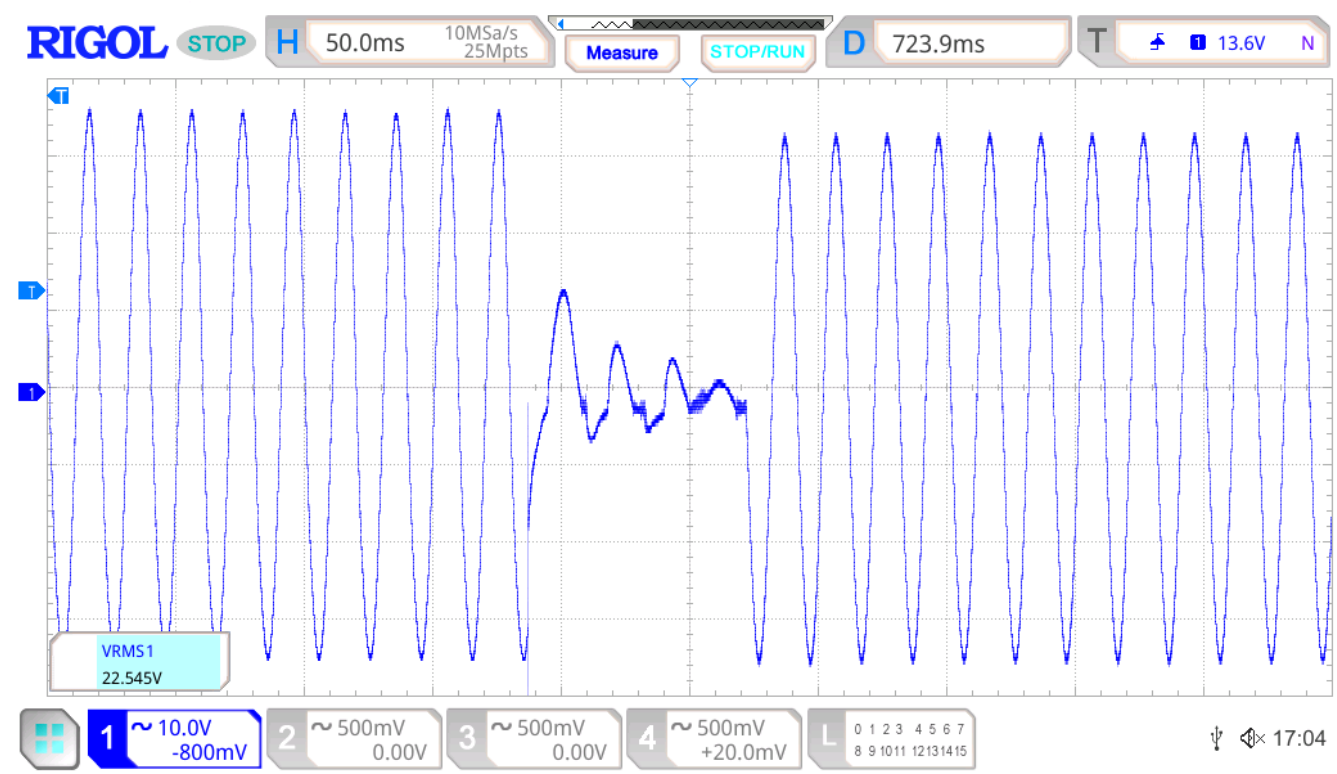

Figure 14. The transition from grid synchronization mode to islanded mode with delay. 


\subsection{Prioritization of the Power Produced by the Inverters}

The purpose of this test is to introduce prioritization of the inverters in parallel work. The main idea behind it is to control the current that is produced by a given inverter to maximize benefits from the current condition of the system that is energy price or the possible amount of energy to be produced from RES. The results of the test are presented in Figure 15. The middle subplot presents changes in current produced by the inverter in grid-following mode. The current reference was changed in steps. The changes in current did not disturb the voltage, neither the frequency.

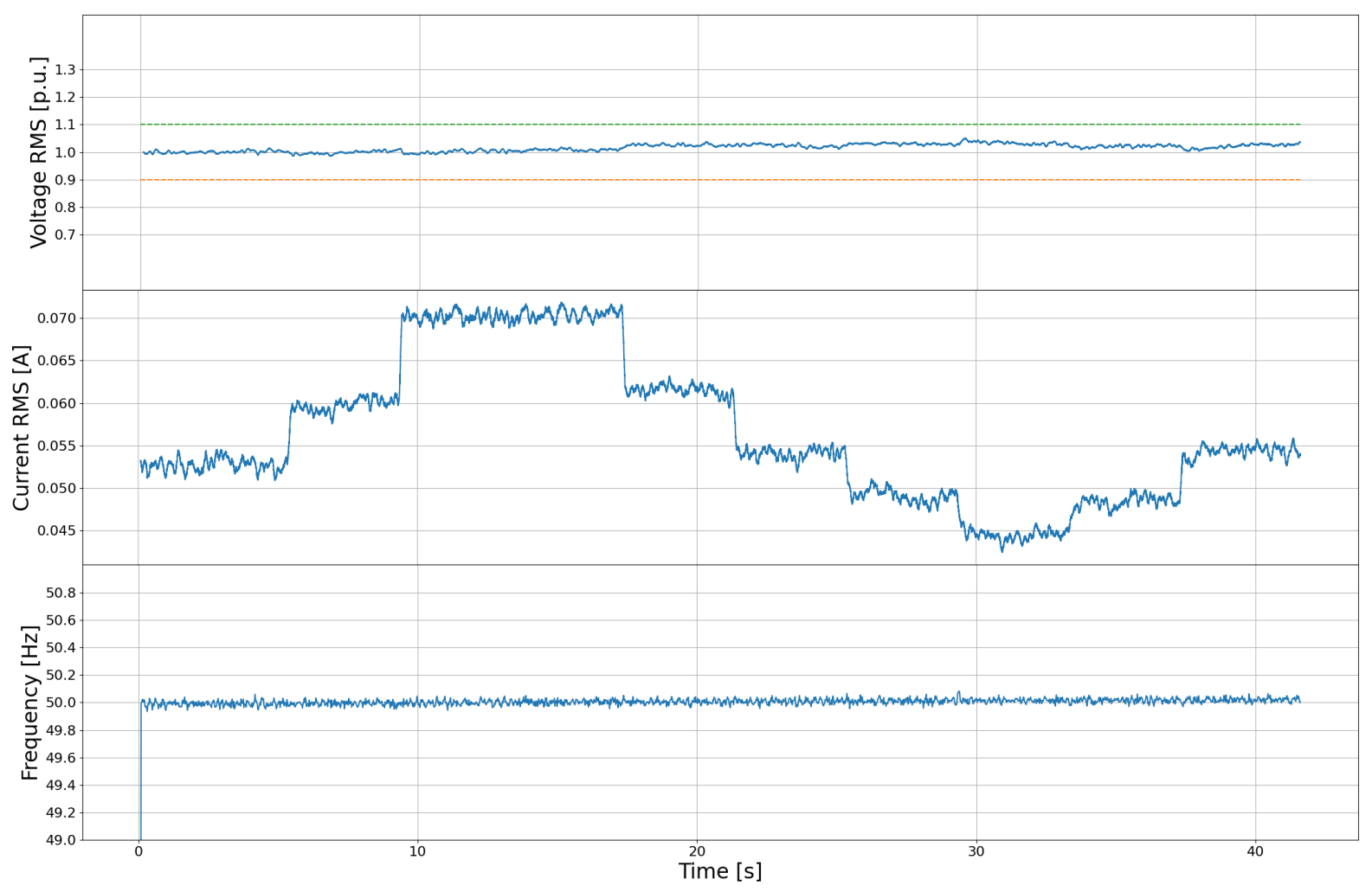

Figure 15. The change of current reference of controlled inverter.

\section{Conclusions}

The paper proposed an inverter-based microgrid energy production emulation for smart grid testing. The focus was on the challenges of parallel energy production under diversity of scenarios in microgrid emulation.

The effectiveness of the response to load changes was investigated. The settling time of DUT was appropriately fast and over- or undershoot was within desired ranges. The ability of connection and removal of a specified number of inverters was tested. Satisfactory results were obtained when the number of parallel working units was changed by one, sufficient results were achieved when the number was changed by two. When the working microgrid consisting of two inverters and another microgrid consisting of four inverters were turned on, the voltage spike ranges defined by standards were violated, thus other supplementary devices should be built-in. The method used to sense grid fault detection and afterward transition to islanding operation works correctly. After about two periods of $50 \mathrm{~Hz}$ the sinusoidal waveforms of output voltage, the inverter remodels the operating state from grid-tied to islanding without voltage spikes or overshoot. Moreover, delay extending the beginning of transition was implemented for safety purposes. The single inverter of the emulator has the ability to control the current that it is producing when the MG consists of more than one inverter. 
The test results for the scenarios evaluated in the paper confirmed the validity of using an inverter in the emulator. An in-depth test procedure revealed the capabilities and possible limitations of using this inverter. Having a good representation of the behavior of the power electronic devices in cooperation with the rest of the emulator will allow in the future to implement additional layers of microgrid management.

\section{Future Work}

Future research will be devoted to the developments towards testing the emulator as a whole, considering all the levels of the electrical system from primary substation through secondary substations down to the prosumer blocks. Further developments and tests are planned to support the grid resilience aspects, like active and reactive power control or voltage and frequency ride-through capabilities. On top of the emulator energy management algorithms will be deployed and evaluated. Another goal of the emulator is to investigate the accurate behavior of energy end users and the information exchange between different actors in the grid.

Author Contributions: Conceptualization, M.K. and K.P.; methodology, M.K. and K.P.; software, M.K.; validation, M.K., K.P. and K.T.; formal analysis, M.K., K.P. and K.T.; investigation, M.K.; resources, M.K., K.P. and K.T.; data curation, M.K.; writing-original draft preparation, M.K., K.P. and K.T.; writing-review and editing, M.K. and K.P.; visualization, M.K. and K.P.; supervision, K.P.; project administration, M.K. and K.P.; funding acquisition, K.P. All authors have read and agreed to the published version of the manuscript.

Funding: This research was funded by EBALANCE-PLUS grant number 864283 .

Institutional Review Board Statement: Not applicable.

Informed Consent Statement: Not applicable.

Data Availability Statement: Not applicable.

Conflicts of Interest: The authors declare no conflict of interest.

\section{References}

1. Deppermann, A.; Paroussos, L.e.a. EU Reference Scenario 2020; Technical Report; European Commission: Brussels, Belgium, 2021.

2. Buchholz, B.; Styczynski, Z. Smart Grids: Fundamentals and Technologies in Electric Power Systems of the Future; Springer: Berlin, Germany, 2020. [CrossRef]

3. Vadari, M. Smart Grid Redefined: Transformation of the Electric Utility; Artech House Titles in Power Engineering; Artech House: Boston, MA, USA, 2018.

4. Olivares, D.E.; Mehrizi-Sani, A.; Etemadi, A.H.; Cañizares, C.A.; Iravani, R.; Kazerani, M.; Hajimiragha, A.H.; Gomis-Bellmunt, O.; Saeedifard, M.; Palma-Behnke, R.; et al. Trends in Microgrid Control. IEEE Trans. Smart Grid 2014, 5, 1905-1919. [CrossRef]

5. Alatrash, H.; Mensah, A.; Mark, E.; Amarin, R.; Enslin, J. Generator Emulation Controls for photovoltaic inverters. In Proceedings of the 2011 14th European Conference on Power Electronics and Applications, Birmingham, UK, 30 August-1 September 2011; pp. 1-10. [CrossRef]

6. Wei, C.; Shen, Z.; Xiao, D.; Wang, L.; Bai, X.; Chen, H. An optimal scheduling strategy for peer-to-peer trading in interconnected microgrids based on RO and Nash bargaining. Appl. Energy 2021, 295, 117024. [CrossRef]

7. Hosseinzadeh, M.; Rajaei Salmasi, F. Islanding Fault Detection in Microgrids-A Survey. Energies 2020, 13, 3479. [CrossRef]

8. IEEE. IEEE Standard for Interconnection and Interoperability of Distributed Energy Resources with Associated Electric Power Systems Interfaces. In IEEE Std 1547-2018 (Revision of IEEE Std 1547-2003); IEEE: New York, NY, USA, 2018; pp. 1-138. [CrossRef]

9. Khadem, M.S.K.; Basu, M.; Conlon, M. Parallel operation of inverters and active power filters in distributed generationsystem-A review. Renew. Sustain. Energy Rev. 2011, 15, 5155-5168. [CrossRef]

10. Han, H.; Hou, X.; Yang, J.; Wu, J.; Su, M.; Guerrero, J.M. Review of Power Sharing Control Strategies for Islanding Operation of AC Microgrids. IEEE Trans. Smart Grid 2016, 7, 200-215. [CrossRef]

11. Vijay, A.S.; Doolla, S.; Chandorkar, M.C. Real-Time Testing Approaches for Microgrids. IEEE J. Emerg. Sel. Top. Power Electron. 2017, 5, 1356-1376. [CrossRef]

12. García-Martínez, E.; Sanz, J.F.; Muñoz-Cruzado, J.; Perié, J.M. A Review of PHIL Testing for Smart Grids—Selection Guide, Classification and Online Database Analysis. Electronics 2020, 9, 382. [CrossRef]

13. Strasser, T.; de Jong, E.; Sosnina, M. European Guide to Power System Testing: The ERIGrid Holistic Approach for Evaluating Complex Smart Grid Configurations; Springer: Cham, Switzerland, 2020. [CrossRef] 
14. Vijay, A.S.; Doolla, S.; Chandorkar, M.C. A Real-time de-risked Emulation based Testing Platform for AC Microgrids. In Proceedings of the 2020 IEEE Energy Conversion Congress and Exposition (ECCE), Detroit, MI, USA, 11-15 October 2020; pp. 2480-2485. [CrossRef]

15. Akdogan, M.E.; Ahmed, S. Control Hardware-in-the-loop for Voltage Controlled Inverters with Unbalanced and Non-linear Loads in Stand-alone Photovoltaic (PV) Islanded Microgrids. In Proceedings of the 2020 IEEE Energy Conversion Congress and Exposition (ECCE), Detroit, MI, USA, 11-15 October 2020; pp. 2431-2438. [CrossRef]

16. Prabakar, K.; Valibeygi, A.; Konakalla, S.A.R.; Miller, B.; De Callafon, R.A.; Pratt, A.; Symko-Davies, M.; Bialek, T. Remote Hardware-in-the-Loop Approach for Microgrid Controller Evaluation. In Proceedings of the 2020 Clemson University Power Systems Conference (PSC), Clemson, SC, USA, 10-13 March 2020; pp. 1-8. [CrossRef]

17. Lauss, G.F.; Faruque, M.O.; Schoder, K.; Dufour, C.; Viehweider, A.; Langston, J. Characteristics and Design of Power Hardwarein-the-Loop Simulations for Electrical Power Systems. IEEE Trans. Ind. Electron. 2016, 63, 406-417. [CrossRef]

18. Nzimako, O.; Wierckx, R. Stability and accuracy evaluation of a power hardware in the loop (PHIL) interface with a photovoltaic micro-inverter. In Proceedings of the IECON 2015-41st Annual Conference of the IEEE Industrial Electronics Society, Yokohama, Japan, 9-12 November 2015; pp. 5285-5291. [CrossRef]

19. Davidson, M.; Abbood, H.D.; Benigni, A. Power Hardware in the Loop testing of a PV micro-inverter. In Proceedings of the 2017 6th International Conference on Clean Electrical Power (ICCEP), Santa Margherita Ligure, Italy, 27-29 June 2017; pp. 145-151. [CrossRef]

20. Wang, Y.; Syed, M.H.; Guillo-Sansano, E.; Xu, Y.; Burt, G.M. Inverter-Based Voltage Control of Distribution Networks: A Three-Level Coordinated Method and Power Hardware-in-the-Loop Validation. IEEE Trans. Sustain. Energy 2020, 11, $2380-2391$. [CrossRef]

21. Prieto-Araujo, E.; Olivella-Rosell, P.; Cheah-Mañe, M.; Villafafila-Robles, R.; Gomis-Bellmunt, O. Renewable energy emulation concepts for microgrids. Renew. Sustain. Energy Rev. 2015, 50, 325-345. [CrossRef]

22. Akbarian, H.; Pillay, P.; Lopes, L. Design of a power electronic emulator for parallel operation of renewable energy resources in microgrids. In Proceedings of the 2015 IEEE International Electric Machines Drives Conference (IEMDC), Coeur d'Alene, ID, USA, 10-13 May 2015; pp. 1532-1537. [CrossRef]

23. Piotrowski, K.; Sojka-Piotrowska, A.; Stamenkovic, Z.; Kraemer, R. IHP Node-The Experimental Platform for Wireless Sensor Networks and Internet of Things . In Proceedings of the 24th Telecommunications Forum (TELFOR), Belgrade, Serbia, 22-23 November 2016. [CrossRef]

24. Piotrowski, K.; Sojka-Piotrowska, A. IHP Node Platform as a Base for Precision Farming and Remote Diagnosis in Agriculture. In Proceedings of the 11th Scientific Conference on Measurement Systems in Research and in Industry, Beijing, China, 20-22 April 2016.

25. Chen, J.; Cañete-Carmona, E.; Garrido, D.; Díaz, M.; Piotrowski, K. PICO: A platform independent communications middleware for heterogeneous devices in smart grids. Comput. Stand. Interfaces 2019, 65. [CrossRef]

26. Piotrowski, K.; Peralta, J.J.; Jiménez-Redondo, N.; Matusiak, B.E.; Zieliński, J.; Casaca, A.; Ciemniewski, W.; Krejtz, K.; Kowalski, J. How to Balance the Energy Production and Consumption in Energy Efficient Smart Neighbourhood; MedPower: Athens, Greece, 2014. [CrossRef]

27. Krysik, M. Control System of the Inverter Model with Regulated Maximum Power and Synchronization with the Power Grid. Master's Thesis, University of Zielona Góra: Zielona Góra, Poland, 2020.

28. Teodorescu, R.; Liserre, M.; Rodriguez, P. Frontmatter. In Grid Converters for Photovoltaic and Wind Power Systems; John Wiley \& Sons: Hoboken, NJ, USA, 2007; pp. i-xvii. [CrossRef]

29. Pattabiraman, D.; Lasseter, R.H.; Jahns, T.M. Comparison of Grid Following and Grid Forming Control for a High Inverter Penetration Power System. In Proceedings of the 2018 IEEE Power Energy Society General Meeting (PESGM), Portland, OR, USA, 5-9 August 2018; pp. 1-5. [CrossRef]

30. Texas Instruments. TMS320F2803x Piccolo, Technical Reference Manual; Texas Instruments Incorporated : Dallas, TX, USA, 2018.

31. Texas Instruments. INA240 High- and Low-Side, Bidirectional, Zero-Drift, Current-Sense Amplifier With Enhanced PWM Rejection; Texas Instruments Incorporated : Dallas, TX, USA, 2018.

32. Kim, M.S.; Haider, R.; Cho, G.J.; Kim, C.H.; Won, C.Y.; Chai, J.S. Comprehensive Review of Islanding Detection Methods for Distributed Generation Systems. Energies 2019, 12, 837. [CrossRef] 